\title{
Correction to: CRMPSoC: New Solution for Feasible Reconfigurable MPSoC
}

Imen Khemaissia, Olfa Mosbahi, Mohamed Khalgui, and Zhiwu Li

\section{Correction to: \\ Chapter "CRMPSoC: New Solution for Feasible \\ Reconfigurable MPSoC" in: E. Cabello et al. (Eds.): \\ Software Technologies, CCIS 743, https://doi.org/10.1007/978-3-319-62569-0_9}

The original version of the chapter "CRMPSoC: New Solution for Feasible Reconfigurable MPSoC", starting on p.175 was revised. An affiliation has been added. The original chapter was corrected. 